\title{
GROUPS OF PERMUTATION PROJECTIVE DIMENSION TWO
}

JAMES E. ARNOLD, JR.

\begin{abstract}
In previous papers we developed a version of homological algebra for $Z[G]$ modules ( $G$ a finite group) using summands of permutation modules in place of projective modules. The resulting theory is more discriminating than the usual homological algebra. For example, an f.g. Z-torsion free $Z[G]$ module is either projective or has infinite projective dimension, whereas for $G$ cyclic, all f.g. $Z[G]$ modules have permutation projective dimension one. In this paper we apply results of Endo and Miyata on permutation projective modules to characterize groups of dimension two.
\end{abstract}

1. Homological algebra based on permutation modules. In this section we summarize the terminology and basic results from [2] (see also [1]).

Definition 1.1. Let $M$ be an f.g. $Z$-free $Z[G]$ module. Then

(a) $M$ is a permutation module if $M$ has a $Z$-basis which is permuted by $G$;

(b) $M$ is a $\mathscr{C}_{1}$ module (or a permutation projective module) if $M$ is a summand of a permutation module.

Note that a permutation module is a sum of modules of the form $Z[G / H]$ for various subgroups $H \subset G$. In particular, it follows that sums of projective modules over quotients of $G$ are $\mathscr{C}_{1}$ modules.

Definition 1.2. Given $H \subset G$ and a $Z[G]$ module $M$, we let $M^{H}=\{x \in M \mid h(x)$ $=x$ for all $h \in H\}$.

The following stronger form of exactness is used to insure that resolutions by $\mathscr{C}_{1}$ modules are well defined up to chain equivalence.

Definition 1.3. An exact sequence $0 \rightarrow A \rightarrow B \rightarrow C \rightarrow 0$ is $H^{0}$ exact if $0 \rightarrow A^{H}$ $\rightarrow B^{H} \rightarrow C^{H} \rightarrow 0$ is exact for all $H \subset G$. A chain complex $C_{*}$ is $H^{0}$ exact if $C_{*}$ and $C_{*}^{H}$ are acyclic for all $H \subset G$.

REMARK. The $H^{0}$ above refers to Hochschild cohomology. Alternatively, the condition we need is right exactness under the Tate $\hat{H}^{0}$. Except in this context, $H^{n}$ will denote the $n$th Tate cohomology group.

Note that (see [2]) $H^{1}(H, A)=0$ for all $H \subset G$ if and only if all short exact sequences with kernel $A$ are $H^{0}$ exact. Also, $H^{0}$ exact sequences with $\mathscr{C}_{1}$ cokernel split. In [2], $\mathscr{C}_{1}-H^{0}$ exact resolutions were constructed for f.g. $Z[G]$ modules, and theorems analogous to those of projective resolutions were established. In particular, we note the following result from which uniqueness of resolutions up to chain equivalence is immediate.

Received by the editors January 23, 1981 and, in revised form, September 2, 1982.

1980 Mathematics Subject Classification. Primary 18G20, 18H10, 20 J05.

1984 American Mathematical Society $0002-9939 / 84 \$ 1.00+\$ .25$ per page 
THEOREM 1.4. Let $\cdots \rightarrow C_{n} \rightarrow C_{n-1} \rightarrow \cdots \rightarrow C_{0} \rightarrow B \rightarrow 0$ be an $H^{0}$ exact sequence, and $\cdots \rightarrow P_{n} \rightarrow P_{n-1} \rightarrow \cdots \rightarrow P_{0} \rightarrow A \rightarrow 0$ an exact sequence with $P_{i} a$ $\mathscr{C}_{1}$ module for all $i$. Then given $f: A \rightarrow B$, there is a chain map $F_{*}: P_{*} \rightarrow C_{*}$ over $f$, and any two such chain maps are homotopic.

The $\mathscr{C}_{1}$ (or permutation projective) dimension of a module is defined as follows:

Definition 1.5. An f.g. $Z[G]$ module $M$ has $\mathscr{C}_{1}$ dimension $\leqslant n$ if $M$ has a $\mathscr{C}_{1}-H^{0}$ exact resolution $P_{*}$ with $P_{n}=0$ for $k>n$. The least such integer $n$ is the $\mathscr{C}_{1}$ dimension of $M$.

As in the projective theory, $\operatorname{dim}(M) \leqslant n$ if and only if the $n$th kernel in a $\mathscr{C}_{1}-H^{0}$ exact resolution of $M$ is a $\mathscr{C}_{1}$ module. In [2] we showed that cyclic groups have $\mathscr{C}_{1}$-global dimension one, and that global dimension one characterizes cyclic groups among the nilpotent groups. More generally, Endo and Miyata have shown (see [4]) that a $Z[G]$ module $M$ is $\mathscr{C}_{1}$ if and only if $M$ is $\mathscr{C}_{1}$ over the Sylow subgroups of $G$. It follows that the global dimension of a group is the maximum global dimension of its Sylow subgroups. In particular, the argument in [1] shows that $G$ has global dimension one if and only if all Sylow subgroups of $G$ are cyclic.

2. Groups of $\mathscr{C}_{1}$-global dimension two. If $M$ is a $\mathscr{C}_{1}$ module, then $H^{1}(H, M)=$ $H^{-1}(H, M)=0$ for all $H \subset G$. The question of whether or not the converse holds was raised by H. W. Lenstra, Jr. Endo and Miyata (see [5]) obtained the following complete solution to Lenstra's problem:

THEOREM 2.1 (ENDO-MIYATA). The following two conditions are equivalent for finite groups $G$ :

(1) $A$ Z-free f.g. $Z[G]$ module $M$ is $\mathscr{C}_{1} \Leftrightarrow H^{1}(H, M)=H^{-1}(H, M)=0$ for all subgroups $H \subset G$.

(2) The p-Sylow subgroups of $G$ are cyclic for p-odd, and the 2-Sylow subgroups are either cyclic or dihedral (including the Klein four group).

Before Endo related the preceding theorem, we had used $H^{0}$ exact sequences and our results for cyclic groups to show that the Klein four group has dimension two. A similar inductive argument works to get from the Klein four group to the dihedral group of order eight. The general inductive step however, seems more complicated than applying Theorem 2.1 as we do here. We will show that the groups in Theorem 2.1 are exactly those of dimension two or less.

In what follows we let $N=\sum_{g \in G} g$ and $I(G)$ be the augmentation ideal of $Z[G]$. $H^{n}(G, A)$ will denote the $n$th Tate cohomology group of $G$ with coefficients in $A$. In particular, $H^{0}(G, A)=A^{G} / N \cdot A$ and $H^{-1}(G ; A)=A_{N} / I(G) \cdot A$ where $A^{G}=\{x \in$ $A \mid g \cdot x=x$ for all $g \in G\}$ and $A_{N}=\{x \in A \mid N \cdot x=0\}$. Note again that when we say a sequence is $H^{0}$ exact we are referring to the Hochschild $H^{0}$.

We let $A^{*}=\operatorname{Hom}_{Z}(A, Z)$ with $(g \cdot f)(x)=f\left(g^{-1} \cdot x\right)$ for $g \in G$ and $f \in A^{*}$. If $A$ has a finite $Z$-basis, then the matrix for $g \in G$ in the dual basis for $A^{*}$ is the inverse transpose of the matrix for $g$ acting on $A$. If $A$ is a permutation module, we can choose a basis permuted by $G$, and it follows that $A^{*}=A$ since the matrices are permutation matrices. 
For our main result we need the fact that $H^{n}(G, A) \simeq H^{-n}\left(G, A^{*}\right)$ when $A$ is f.g. $Z$-free. This duality can be obtained from Proposition (6.2) in Chapter 12 of Cartan and Eilenberg (see [3]), but we include a short proof here since we were unable to find this result explicitly in the literature.

Theorem 2.2. Let $A$ be a $Z$-free f.g. $Z[G]$ module. Then $H^{n}(G, A) \simeq H^{-n}\left(G, A^{*}\right)$.

Proof. For $n=0$, we have $H^{0}\left(G, A^{*}\right)=\left(A^{*}\right)^{G} / N \cdot A^{*}$.

$$
\left(A^{*}\right)^{G}=\left\{f \in A^{*} \mid f(I(G) \cdot A)=0\right\}=\left\{f \in A^{*} \mid f\left(A_{N}\right)=0\right\}
$$

since $A_{N} / I(G) \cdot A$ is all $|G|$-torsion. Thus we can identify $\left(A^{*}\right)^{G}$ with $\operatorname{Hom}_{Z}\left(A / A_{N}, Z\right) \simeq \operatorname{Hom}(N \cdot A, Z)$, and under the canonical isomorphism $N \cdot A^{*}$

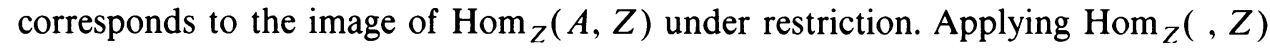
to the exact sequence

$$
0 \rightarrow N \cdot A \rightarrow A \rightarrow A / N \cdot A \rightarrow 0,
$$

it follows that $H^{0}\left(G, A^{*}\right) \simeq \operatorname{Ext}_{Z}^{1}(A / N \cdot A, Z)$. Therefore, since the torsion part of $A / N \cdot A$ is $H^{0}(G, A)$, we have $H^{0}\left(G, A^{*}\right) \simeq \operatorname{Ext}_{Z}^{1}\left(H^{0}(G, A), Z\right) \simeq H^{0}(G, A)$. For $n>0$, let $K_{n}$ be the $n$th kernel of a projective resolution of $A^{*}$. Then $H^{-n}\left(G, A^{*}\right) \simeq$ $H^{0}\left(G, K_{n}\right)$, and dualizing, $A$ is the $n$th kernel of a projective resolution of $K_{n}^{*}$; hence $H^{n}(G, A) \simeq H^{0}\left(G, K_{n}^{*}\right)$. From the $n=0$ case we have

$$
H^{-n}\left(G, A^{*}\right) \simeq H^{0}\left(G, K_{n}\right) \simeq H^{0}\left(G, K_{n}^{*}\right) \simeq H^{n}(G, A)
$$

for all $n>0$, and since $A=\left(A^{*}\right)^{*}$ we have the result for all integers $n$.

The construction in the next lemma is useful in producing sequences with \pm 1 cohomology zero.

LEMMA 2.3. Let $A$ be an $f$.g. Z-free $Z[G]$ module with $H^{1}(H, A)=0$ for all $H \subset G$. There is an exact sequence of the form $0 \rightarrow P_{1} \rightarrow K \oplus P_{0} \rightarrow A \rightarrow 0$ where $P_{i}$ is a permutation module $i=0,1$, and $H^{ \pm 1}(H, K)=0$ for all $H \subset G$.

Proof. Let $0 \rightarrow B \rightarrow P_{0} \rightarrow A \rightarrow 0$ be $H^{0}$ exact, $P_{0}$ a permutation module. Then choose $0 \rightarrow K_{1} \rightarrow P_{1} \rightarrow B^{*} \rightarrow 0$ exact with $P_{1}$ a permutation module. Now consider the following commutative diagram:

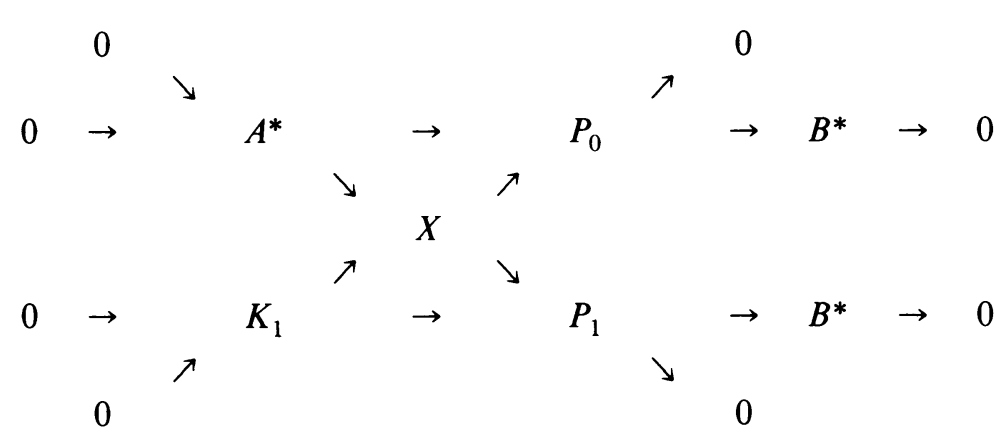


The first sequence is the dual of $0 \rightarrow B \rightarrow P_{0} \rightarrow A \rightarrow 0$, and $X$ is the pullback obtained from the diagram:

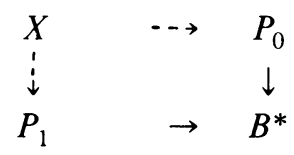

The sequences with $X$ in the middle we exact, and are obtained by projecting $X$ on $P_{0}$ and $P_{1}$ respectively. Now since $0 \rightarrow K_{1} \rightarrow P_{1} \rightarrow B^{*} \rightarrow 0$ is $H^{0}$ exact, $H^{1}\left(H, K_{1}\right)$ $=0$ for all $H \subset G$. Therefore $0 \rightarrow K_{1} \rightarrow X \rightarrow P_{0} \rightarrow 0$ is also $H^{0}$ exact and splits since $P_{0}$ is a permutation module. Replacing $X$ with $P_{0} \oplus K_{1}$ in the other sequence we get

$$
0 \rightarrow A^{*} \rightarrow P_{1} \oplus K_{1} \rightarrow P_{1} \rightarrow 0
$$

exact. Since $H^{1}\left(H, A^{*}\right)=0$ for all $H \subset G$, we have $H^{-1}\left(H, A^{*}\right)=0$ for all $H \subset G$ by Theorem 2.2. The long exact cohomology sequence given by $(2.5)$ is

$$
\cdots \rightarrow H^{-1}\left(H, A^{*}\right) \rightarrow H^{-1}\left(H, P_{0}\right) \oplus H^{-1}\left(H, K_{1}\right) \rightarrow H^{-1}\left(H, P_{1}\right) \rightarrow \cdots
$$

at -1 . Therefore since $H^{-1}\left(H, P_{i}\right)=0$ for all $H \subset G$, we have $H^{-1}\left(H, K_{1}\right)=0$ for all $H \subset G$. Now dualizing (2.5) we have

$$
0 \rightarrow P_{1} \rightarrow P_{0} \oplus K_{1}^{*} \rightarrow A \rightarrow 0
$$

and $H^{ \pm 1}\left(H, K_{1}^{*}\right)=0$ for all $H \subset G$. Letting $K=K_{1}^{*}$ we have the lemma.

The following theorem together with Endo and Miyata's Theorem will characterize groups of dimension one and two.

THEOREM 2.6. For a finite group $G$ the following are equivalent:

(1) The $\mathscr{C}_{1}$ global dimension of $G$ is two or less.

(2) $A$ Z-free f.g. $Z[G]$ module $A$ is $\mathscr{C}_{1} \Leftrightarrow H^{ \pm 1}(H, A)=0$ for all subgroups $H \subset G$.

Proof. (1) $\Rightarrow(2)$ : If $G$ has dimension two or less, and $A$ is $Z$-free f.g. with $H^{ \pm 1}(H, A)=0$ for all $H \subset G$, then choose an exact sequence $0 \rightarrow A \rightarrow P \rightarrow M \rightarrow 0$ with $P$ a projective module. This is $H^{0}$ exact since $H^{1}(H, A) \equiv 0$. Since $\operatorname{dim}(M) \leqslant 2$ there is an exact sequence $0 \rightarrow K_{1} \rightarrow P_{1} \rightarrow A \rightarrow 0$ with $P_{1}$ a permutation module and $K_{1}$ a $\mathscr{C}_{1}$ module. Dualizing we get $0 \rightarrow A^{*} \rightarrow P_{1} \rightarrow K_{1}^{*} \rightarrow 0$ and $H^{ \pm 1}\left(H, A^{*}\right)$ $\equiv 0$ by Theorem 2.2. Now since $H^{1}\left(H, A^{*}\right) \equiv 0$, the sequence is $H^{0}$ exact, and splits since $K_{1}^{*}$ is $\mathscr{C}_{1}$. Therefore $A^{*}$ is $\mathscr{C}_{1}$ and hence $A$ is also $\mathscr{C}_{1}$.

(2) $\Rightarrow$ (1): Given an f.g. $Z[G]$ module $M$, choose $0 \rightarrow A \rightarrow P \rightarrow M \rightarrow 0 H^{0}$ exact with $P$ a permutation module. Since $H^{1}(H, A)=0$ for all $H \subset G$, there is (by 2.3) an exact sequence $0 \rightarrow P_{1} \rightarrow K \oplus P_{0} \rightarrow A \rightarrow 0$ with $P_{i}$ permutation modules $i=1,0$ and $H^{ \pm 1}(H, K) \equiv 0$. By (2), $K$ is a $\mathscr{C}_{1}$ module, and splicing this sequence with the first sequence at $A$, we have $\operatorname{dim}(M) \leqslant 2$.

The following corollary is now immediate from Endo and Miyata's Theorem.

COROLlary 2.7. The $\mathscr{C}_{1}$ global dimension of $G$ is two if and only if the odd p-Sylow subgroups of $G$ are cyclic, and $G$ has a dihedral 2-Sylow subgroup. 
In conclusion, we note that whenever the dimension of $G$ is finite, $G_{0}(Z[G])$ is isomorphic to the Grothendieck group with $\mathscr{C}_{1}$ modules as generators, and relations $\Sigma(-1)^{i} P_{i}=0$ for each exact sequence $0 \rightarrow P_{n} \rightarrow \cdots \rightarrow P_{0} \rightarrow 0$ of $\mathscr{C}_{1}$ modules (see [1]). Thus, for the groups in Theorem 2.1, the generalized Cartan homomorphism (defined in [1]) from the above Grothendieck group to $G_{0}(Z[G])$ is an isomorphism.

\section{REFERENCES}

1. J. E. Arnold, Jr., A generalized Cartan isomorphism for the Grothendieck group of a finite group, J. Pure Appl. Algebra 12 (1978), 225-234.

2. Homological algebra based on permutation modules, J. Algebra 70 (1981), 250-260.

3. H. Cartan and S. Eilenberg, Homological algebra, Princeton Univ. Press, Princeton, N. J., 1956.

4. S. Endo and T. Miyata, On a classification of the function fields of algebraic tori, Nagoya Math. J. 56 (1975), 85-104.

5. __ Integral representations with trivial first cohomology groups, preprint.

Department of Mathematical Sciences, University of Wisconsin - Milwaukee, Milwaukee, WISCONSIN 53201 